\title{
CT-based Radiomics Combined with Signs: A Valuable Tool to help Physician Discriminate COVID- 19 and Other Viral Pneumonia
}

\section{Yilong Huang}

Medical Imaging Department $\bigotimes$ First Affiliated Hospital of Kunming Medical University

\section{Zhenguang Zhang}

Medical Imaging Department, First Affiliated Hospital of Kunming Medical University

\section{Xiang Li}

Radiology Department囚Third People's Hospital of Kunming

\section{Yunhui Yang}

Medical Imaging Department $₫$ People's Hospital of Xishuangbanna Dai autonamous prefecture

\section{Zhipeng Li}

Medical Imaging Department囚Yunnan Provincial Infectious Disease Hospital

\section{Jialong Zhou}

MRI Department囚First People's Hospital of Yunnan

\section{Yuanming Jiang}

Medical Imaging Department $\triangle$ First Affiliated Hospital of Kunming Medical University

\section{Jiyao Ma}

Medical Imaging Department\First Affiliated Hospital of Kunming Medical University

\section{Siyun Liu}

GE Healthcare Life Sciences

Bo HE ( $\sim$ henri5437@sina.com )

First Affiliated Hospital of Kunming Medical University https://orcid.org/0000-0002-0818-9448

\section{Research}

Keywords: coronavirus disease 2019, viral pneumonia, radiomics, X-ray computed tomography

Posted Date: August 24th, 2020

DOI: https://doi.org/10.21203/rs.3.rs-63088/v1

License: (1) (1) This work is licensed under a Creative Commons Attribution 4.0 International License.

Read Full License 


\section{Abstract}

Background: In this COVID-19 pandemic, the differential diagnosis of different viral types of pneumonia is still challenging. We aimed to assess the classification performance of computed tomography (CT)based CT signs and radiomics features for discriminating COVID-19 pneumonia and other viral pneumonia.

Methods: A total of 181 patients with confirmed viral pneumonia (COVID-19: 89 cases, Non-COVID-19: 92 cases; training cohort: 126 cases; test cohort: 55 cases) were collected retrospectively in this study. Pneumonia signs and radiomics features were extracted from the initial unenhanced chest CT images to build independent and combined models. The predictive performance of the radiomics model and the combined model were evaluated using an intra-cross validation cohort. Diagnostic performance of two models was assessed via receiver operating characteristic (ROC) analysis.

Results: The combined models consisted of 3 significant CT signs and 14 selected features and demonstrated better discrimination performance between COVID-19 and Non-COVID-19 pneumonia than the single radiomics model. For the radiomics model along, the area under the ROC curve (AUC) were 0.904 (sensitivity, 85.5\%; specificity, 84.4\%; accuracy, 84.9\%) in the training cohort and 0.866 (sensitivity, $77.8 \%$; specificity, 78.6\%; accuracy, 78.2\%) in the test cohort. After combining CT signs and radiomics features, AUC of the combined model for the training cohort was 0.956 (sensitivity, $91.9 \%$; specificity, $85.9 \%$; accuracy, $88.9 \%$ ), while that for the test cohort was 0.943 (sensitivity, $88.9 \%$; specificity, $85.7 \%$; accuracy, $87.3 \%)$.

Conclusion: CT-based radiomics combined with signs might be a potential method for distinguishing COVID-19 and other viral pneumonia with satisfactory performance.

\section{Background}

In December 2019, a highly infectious disease caused by severe acute respiratory syndrome coronavirus 2 (SARS-CoV-2) infection broke out in Wuhan, China, known as Coronavirus disease 2019 (COVID-19) [1, 2]. COVID-19 is spreading around the world at an alarming rate. As of May 9th, more than 3.9 million patients have been diagnosed with COVID-19 with more than 270,000 deaths. Early studies have shown that almost all COVID-19 patients have pneumonia ${ }^{[3,4]}$. However, pneumonia caused by other viral infections is also very common at the same period of year, and clinical symptoms are very similar ${ }^{[5-7]}$. Therefore, in this COVID-19 pandemic, the differential diagnosis of different viral types of pneumonia is difficult but highly important. Real-time reverse transcription-polymerase chain reaction (RT-PCR) is the gold standard for the diagnosis of viral pneumonia. However, recent reports have shown that RT-PCR detection of COVID-19 has low sensitivity ${ }^{[8]}$, and the high false-negative rate limits the rapid identification of viral pneumonia by RT-PCR. 
Currently, computed tomography (CT) can play an important role in the diagnosis and treatment of viral pneumonia ${ }^{[9,10]}$. Studies have shown that the imaging signs of viral pneumonia and bacterial pneumonia are different ${ }^{[11,12]}$. However, little is known about the differences in imaging signs between COVID-19 and other viral pneumonia. And the radiologist's diagnosis of viral pneumonia through imaging signs is a subjective assessment, and the accuracy of diagnosis depends on the doctor's diagnosis experience. Therefore, it is also necessary to further develop a rapid quantitative auxiliary diagnostic method.

Radiomics is a new quantitative analysis technology based on medical imaging, which could extract thousands imaging features including first-order statistical, shape, second- or higher order texture features. Previous studies have shown that radiomics has outstanding performance in tumor diagnosis, treatment effect evaluation, and prognosis prediction ${ }^{[13-15]}$. Recently, there had already been some constructed radiomics model based on deep-learning to predict the prognosis of COVID-19 patient ${ }^{[16]}$. And Chen et al. found that radiomics model based on CT images is a feasible and promising method for monitoring poor prognostic outcome in patients with COVID-19 ${ }^{[17-18]}$. Besides, radiomics has been also used to identify focal organizing pneumonia and peripheral lung adenocarcinoma ${ }^{[19]}$. Therefore, radiomics may be a potential tool for the doctor to identify COVID-19 and other viral pneumonia.

Therefore, in present study, we aim to select significant chest CT signs and radiomics features that can effectively identify COVID-19 pneumonia and other viral pneumonia, and determine whether a CT-based radiomics signature combined with CT signs could be used as a tool in the differentiation of COVID-19 and other viral pneumonia.

\section{Methods}

\section{Patients}

This retrospective study was approved by our institutional review board and patient consent was waived. This study retrospectively collected patients with viral pneumonia diagnosed in 15 hospitals in this province from March 1, 2015 to March 15, 2020. RT-PCR assays were performed to identify influenza A virus, influenza B virus, respiratory syncytial virus, parainfluenza virus, adenovirus, SARS coronavirus, SARS-CoV-2, Epstein-Barr virus, measles virus, and other viruses from nasopharyngeal swabs or bronchoalveolar lavage fluid. The study only included pneumonia patients infected with single virus, and patients with multiple respiratory viruses or bacterial or fungal infections were excluded. A total of 375 viral pneumonia patients were diagnosed in this study. The further selection process for viral pneumonia patients is shown in Figure 1. Patients completed the chest CT examination within 48 hours after admission. According to the virus type found in the lungs, the patients were divided into two groups: COVID-19 and Non-COVID-19. The number of cases included in each hospital is summarized in Supplementary Table 1. 


\section{CT examination}

HRCT examination: CT scanners with 16 or more detector rows (Siemens, Germany; Philips, the Netherlands; and GE, USA) were used. The patient was scanned in the supine position while holding his or her breath after inspiration. The scanning range was from the thoracic inlet to the costophrenic angles. Scanning parameters: detector collimation width $64 \times 0.6 \mathrm{~mm}$ or $128 \times 0.6 \mathrm{~mm}$, tube voltage $120 \mathrm{kV}$, adaptive tube current, high-resolution algorithm reconstruction, reconstruction layer thickness 1 or 1.5 $\mathrm{mm}$, and layer spacing $1.5 \mathrm{~mm}$.

\section{Chest CT signs analysis}

Three Chinese radiologists were blinded to the RT-PCR results, all patient information, and type of viral pneumonia. First, two experienced radiologists in the cardiothoracic group independently read the radiographs. When their opinions were inconsistent, they discussed them and reached a consensus, which was reviewed and confirmed by the third senior radiologist in the cardiothoracic group. The signs of the first CT examination after admission were analyzed. The CT imaging evaluation included lesion location (left upper lobe, left lower lobe, right upper lobe, right middle lobe and right lower lobe) and signs [GGO (ground-glass opacities), partial consolidation, consolidation (multifocal consolidation, focal consolidation), fibrous stripes, septal thickening, intralobular interstitial thickening, subpleural lines, crazypaving pattern, tree-in-bud, bronchial wall thickening, bronchiectasis, air bronchogram, halo sign, reversed halo sign, mediastinal lymphadenectasis, pleural thickening, and pleural effusion] ${ }^{[9-12,20]}$. The window width and level were set to $1600 /-600 \mathrm{HU}$.

\section{CT image processing and volume of interest (VOI) segmentation}

The Lung Kit software (GE Healthcare, Version LK2.2) was used for pneumonia lesion segmentation. All the $\mathrm{CT}$ images were firstly resampled into isotropic $1 \mathrm{~mm} \times 1 \mathrm{~mm} \times 1 \mathrm{~mm}$ voxel size. The five anatomic lung lobes were firstly automatically segmented. Then pneumonia lesion volume of interest (VOI) was automatically segmented and the margin of the VOI was manually comfirmed by experienced thoracic radiologist. The distributed lesions were considered as a whole VOI in the next analysis steps.

\section{Radiomics feature extraction and selection}

A total of 1316 radiomics features were extracted from segmented VOls by using open source of Python package Pyradiomics ${ }^{[21]}$. The extracted radiomics features were categorized into five groups: (1) Firstorder features including 18 intensity statistics and 14 shape features; (2) 75 multi-dimensional texture features including 24 Gray Level Co-occurrence Matrix (GLCM), 16 Gray Level Size Zone Matrix (GLSZM), 16 Gray Level Run Length Matrix (GLRLM), 14 Gray Level Dependence Matrix (GLDM) and 5 Neighboring 
Gray Tone Difference Matrix (NGTDM) Features; (3)1209 Transformed first-order and textural features including: 744 wavelet features in frequency channels LHL, LLH, HHH, HLH, HLL,HHL, LHH and LLL; 186 LoG filtered features with sigma of 2.0 and 3.0; 279 local binary pattern (LBP) filtered texture features.

The radiomics feature data was firstly preprocessed by replacing missing values with median values, and $z$-score normalization was followed. The whole dataset was randomly divided into training and test cohort at the ratio of 7:3. And the radiomics features in the training set was further screened for classification model construction. Firstly, the redundant collinear features were reduced by correlation analysis at a cut-value of 0.7. Then the features without statistical differences between COVID-19 and Non-COVID-19 groups were excluded by Mann-Whitney $U$ test. The significant level was $p<0.05$. The univariate logistic analysis was used to select the potential classification indicators with $P$ value less than 0.05. Next, the least absolute shrinkage and selection operator (LASSO) logistic regression method with 10-fold cross validation was applied for further feature selection and regularization to improve the model accuracy and avoid overfitting. The minimum mean square error for model fitting among the 10 folds was utilized to determine the optimized lambda values. The remaining features with non-zero coefficients at such lambda values were kept for model construction.

\section{Classification model construction}

The logistic regression model was constructed using the selected radiomics features to differentiate COVID-19 from Non-COVID-19 and the Radscore for each patient was calculated based on the regression coefficients. In addition, the independent predictors among CT signs were also selected by using Chisquare test (or Fisher exact test), univariate and multivariate logistic regression methods. These selected CT signs were further combined with radiomics features to construct combined model using logistic regression method. The nomogram of such combined model was also established.

The radiomics model and combined model constructed based on the training set were validated in the test cohort. The classification performances were evaluated by receiver operating characteristic (ROC) curve. The area under the curve (AUC), accuracy, sensitivity and specificity were derived. In addition, the calibration curves and decision curve analysis (DCA) curves were calculated to assess the models' classification performance and their clinical benefits.

\section{Statistical analysis}

The continuous variables or ordinal variables were compared by t-test or Mann-Whitney U test. The distribution of different CT signs was compared by Chi-squared test or Fisher exact test when small sample sizes existed. For ROC analysis, the cut-off value in the training set at the maximum of Youden index of each model was calculated and the confusion matrix and sensitivity, specificity, accuracy in the training and test cohorts were derived at such cut-off value. The Delong test was used for comparison of ROC curves between different models. The reported statistical significance levels were all two-sided with 
the statistical significance set as $p<0.05$. The statistical analyses were performed with SPSS Software (Version 25, IBM, Chicago, IL) and R software (Version: 3.6.1, https: www.r-project.org). The following R packages were mainly involved including: "glmnet" for logistic regression including LASSO regression; "pROC" for ROC analysis; "rmda" for DCA analysis.

\section{Results}

\section{Clinical data}

This study included 181 patients with viral pneumonia (male: 94 ; female: 87 . Mean age: $44.89 \pm 13.85$ ), including 89 cases of COVID-19 pneumonia and 92 cases of Non-COVID-19 pneumonia (influenza A virus, 42 cases; cytomegalovirus, 20 cases; influenza B virus, 18 cases; Epstein-Barr virus, 4 cases; herpes virus, 3 cases; adenovirus, 2 cases; varicella virus, 2 cases; measles virus, 1 case). There was no significant difference in age or gender between the COVID-19 group and the Non-COVID-19 group ( $p>0.05)$ (Table 1$)$.

Table 1

Basic characteristics in the training and test cohort

\begin{tabular}{|c|c|c|c|c|c|c|}
\hline \multirow[t]{2}{*}{ Sets } & \multirow{2}{*}{$\begin{array}{l}\text { No. of } \\
\text { patients }\end{array}$} & \multicolumn{2}{|c|}{ Types of viral pneumonia } & \multirow[t]{2}{*}{ Age } & \multicolumn{2}{|l|}{ Gender } \\
\hline & & COVID-19 & $\begin{array}{l}\text { Non-COVID- } \\
19\end{array}$ & & Male & Female \\
\hline $\begin{array}{l}\text { Training } \\
\text { cohort }\end{array}$ & 126 & $\begin{array}{l}62 \\
(49.20 \%)\end{array}$ & $64(50.79 \%)$ & $44.32 \pm 12.47$ & $\begin{array}{l}65 \\
(51.59 \%)\end{array}$ & $\begin{array}{l}61 \\
(48.41 \%)\end{array}$ \\
\hline Test cohort & 55 & $\begin{array}{l}27 \\
(49.09 \%)\end{array}$ & $28(50.91 \%)$ & $46.19 \pm 18.26$ & $\begin{array}{l}29 \\
(52.73 \%)\end{array}$ & $\begin{array}{l}26 \\
(47.27 \%)\end{array}$ \\
\hline$p$ & - & 0.989 & & 0.516 & 0.888 & \\
\hline
\end{tabular}

Note: COVID-19, coronavirus disease 2019. Continues data are expressed as mean \pm SD, and categorical data are presented as $\mathrm{n}(\%)$.

\section{Chest CT signs of viral pneumonia and its predictive performance}

The 24 CT signs of COVID-19 and Non-COVID-19 were gradually screened by using Chi-square test (Fisher exact test), univariate and multivariate logistic regression analysis. Three independent predictors were selected, including GGO (Coefficient: 3.2502), intralobular interstitial thickening (Coefficient: 1.6963) and halo sign (Coefficient: 1.1967). Select GGO to build a logistic model and evaluate the performance of the model in the training and verification groups (Table 2). Table 2 shows the performance of the prediction model by using GGO, intralobular interstitial thickening, and halo sign. 
Table 2

Predictive performance of the logistic model of CT signs in the differentiation between COVID-19 and Non-COVID-19.

\begin{tabular}{|lll|}
\hline Parameters & Training cohort & Test cohort \\
\hline AUC $(95 \% \mathrm{Cl})$ & $0.875(0.819,0.932)$ & $0.812(0.698,0.925)$ \\
\hline Sensitivity $(\%, 95 \% \mathrm{Cl})$ & $98.4 \%(0.91,1.00)$ & $92.6 \%(0.76,0.99)$ \\
\hline Specificity $(\%, 95 \% \mathrm{Cl})$ & $56.3 \%(0.44,0.68)$ & $53.6 \%(0.36,0.70)$ \\
\hline Positive prediction $(\%, 95 \% \mathrm{Cl})$ & $68.5 \%(0.58,0.77)$ & $65.8 \%(0.50,0.79)$ \\
\hline Negative prediction $(\%, 95 \% \mathrm{Cl})$ & $97.3 \%(0.85,1.00)$ & $88.2 \%(0.64,0.98)$ \\
\hline Accuracy $(\%, 95 \% \mathrm{Cl})$ & $77.0 \%(0.69,0.84)$ & $72.7 \%(0.60,0.83)$ \\
\hline Precision $(\%, 95 \% \mathrm{Cl})$ & $68.5 \%(0.58,0.77)$ & $65.8 \%(0.50,0.79)$ \\
\hline Note: COVID-19, coronavirus disease $2019 ; \mathrm{AUC}$, area under curve. \\
\hline
\end{tabular}

\section{Extraction and selection of radiomics features and building of the radiomics prediction model}

Features dimension reduction was performed on 1316 radiomics features and 14 reliable imaging features were finally selected to identify COVID-19 pneumonia from other types of viral pneumonia. The regression coefficients of each features in the logistic regression model are plotted in Figure 2 . The statistical difference of each radiomics feature between COVID-19 and Non-COVID-19 are summarized in Supplementary Table 2. The Radscore constructed by the selected radiomics features are as follows:

Radscore $=-0.2993-0.0384$ *wavelet-LHH_glszm_LowGrayLevelZoneEmphasis

-1.3885*|bp-3D-m2_glszm_SmallAreaHighGrayLevelEmphasis

$-0.2096 *$ wavelet-LLH_firstorder_Energy

-0.6803* log-sigma-3-0-mm-3D_firstorder_Range

$+0.1782^{*}$ original_shape_Sphericity

$-0.8760 *$ wavelet-HHH_glcm_InverseVariance

+0.1719* log-sigma-3-0-mm-3D_glszm_ZoneVariance

$-0.4058 *$ Ibp-3D-m2_firstorder_Median

+0.9252* wavelet-HHL_glszm_GrayLevelNonUniformityNormalized 
-1.2080* Ibp-3D-m2_gldm_GrayLevelNonUniformity

$-0.6254 *$ Ibp-3D-k_glszm_GrayLevelNonUniformityNormalized

$+0.0446 *$ wavelet-LHH_glrlm_LongRunHighGrayLevelEmphasis

$-0.8229 *$ log-sigma-2-0-mm-3D_firstorder_Maximum

Based on the radiomics features, three significant CT signs are combined to build a model as follows:

Model_score $=-3.2733+0.987 *$ Radscore $+2.5225 * \mathrm{GGO}+2.2599 *$ Intralobular interstitial thickening $+1.1792 *$ Halo sign

Figure 3 is a nomogram of a prediction model established by combining radiomics features and CT signs. Distributions of the Radscore and types of viral pneumonia in the training cohort and test cohort are shown in Figure 4.

\section{Predictive performance of the radiomics model and combined model}

The radiomics features have good performance in the training and test cohorts (Table 3). After combining the radiomics feature and CT signs, the AUC values of were higher than that of radiomics model alone in both cohorts (Figure $5 a$ and $b$ ). The AUC of the two models was compared by Delong test, and the differences were statistically significant (training cohort $p=0.01506$, test cohort $p=0.01506$ ) which indicated the enhancement of the prediction performance of combined model. The calibration curves of two models demonstrated the COVID-19 prediction probability had a good agreement between the prediction and observation in both training cohort and test cohort (Figure $5 \mathrm{c}$ and d). Moreover, the accuracy, sensitivity, specificity, precision, positive prediction, and negative prediction of radiomics features with CT signs are higher than that of radiomics features (Table 3 ). The wide range of high-risk threshold (0-0.75) of the DCA curves in the combined model also indicated its clinical usefulness with standardized net benefits larger than 0.6 (Figure $5 e$ and $5 f$ ). 
Table 3

Predictive performance of the radiomics model and radiomics with CT signs model

\begin{tabular}{|c|c|c|c|c|}
\hline \multirow[t]{2}{*}{ Parameters } & \multicolumn{2}{|l|}{ Radiomics } & \multicolumn{2}{|c|}{ Radiomics +CT signs } \\
\hline & Training cohort & Test cohort & Training cohort & Test cohort \\
\hline Cutoff-value & $>-0.1$ & $>-0.1$ & $>-0.415$ & $>-0.415$ \\
\hline Accuracy $(\%, 95 \% \mathrm{Cl})$ & $\begin{array}{l}84.9 \%(0.7756 \\
0.9021)\end{array}$ & $\begin{array}{l}78.2 \%(0.6548, \\
0.8720)\end{array}$ & $\begin{array}{l}88.9 \%(0.8210 \\
0.9338)\end{array}$ & $\begin{array}{l}87.3 \%(0.7568, \\
0.9400)\end{array}$ \\
\hline $\begin{array}{l}\text { Sensitivity (\%, } \\
95 \% \mathrm{Cl})\end{array}$ & $\begin{array}{l}85.5 \%(0.7443 \\
0.9240)\end{array}$ & $\begin{array}{l}77.8 \%(0.5890, \\
0.8974)\end{array}$ & $\begin{array}{l}91.9 \%(0.8208 \\
0.9690)\end{array}$ & $\begin{array}{l}88.9 \%(0.7112, \\
0.9697)\end{array}$ \\
\hline $\begin{array}{l}\text { Specificity (\%, } \\
95 \% \mathrm{Cl})\end{array}$ & $\begin{array}{l}84.4 \%(0.7337 \\
0.9148)\end{array}$ & $\begin{array}{l}78.6 \%(0.6011, \\
0.9014)\end{array}$ & $\begin{array}{l}85.9 \%(0.7516 \\
0.9265)\end{array}$ & $\begin{array}{l}85.7 \%(0.6789, \\
0.9492)\end{array}$ \\
\hline $\begin{array}{l}\text { Precision } \\
(\%, 95 \% \mathrm{Cl})\end{array}$ & $\begin{array}{l}84.1 \%(0.7299, \\
0.9134)\end{array}$ & $\begin{array}{l}77.8 \%(0.5890, \\
0.8974)\end{array}$ & $\begin{array}{l}86.4 \%(0.7585 \\
0.9288)\end{array}$ & $\begin{array}{l}85.7 \%(0.6789, \\
0.9492)\end{array}$ \\
\hline $\begin{array}{l}\text { Positive prediction } \\
(\%, 95 \% \mathrm{Cl})\end{array}$ & $\begin{array}{l}84.1 \%(0.7299 \\
0.9134)\end{array}$ & $\begin{array}{l}77.8 \%(0.5890, \\
0.8974)\end{array}$ & $\begin{array}{l}86.4 \%(0.7585 \\
0.9288)\end{array}$ & $\begin{array}{l}85.7 \%(0.6789, \\
0.9492)\end{array}$ \\
\hline $\begin{array}{l}\text { Negative prediction } \\
(\%, 95 \% \mathrm{Cl})\end{array}$ & $\begin{array}{l}85.7 \%(0.7480 \\
0.9252)\end{array}$ & $\begin{array}{l}78.6 \%(0.6011, \\
0.9014)\end{array}$ & $\begin{array}{l}91.7 \%(0.8153, \\
0.9679)\end{array}$ & $\begin{array}{l}88.9 \%(0.7112, \\
0.9697)\end{array}$ \\
\hline
\end{tabular}

\section{Discussion}

Considering the similarity of period of year, clinical symptoms and CT findings of COVID-19 and other viral pneumonia and the importance of differential diagnosis, this study systematically analyzed the differences in imaging signs and radiomics between them. Our research found that three signs and fourteen radiomics features are related to COVID-19 infection. In our study, the diagnostic performance of the radiomics model was better than the radiologists' subjective judgments. Moreover, the combined model was based on CT signs and radiomics features, which can distinguish COVID-19 from other viral pneumonia well and show excellent and encouraging performance.

This study included 181 confirmed patients with viral pneumonia, and we compared the CT signs of COVID-19 and other viral pneumonia. We found that GGO, intralobular interstitial thickening and halo sign of COVID-19 pneumonia are more common than other viral pneumonia, which is consistent with previous studies ${ }^{[20,22]}$. And the performance of CT signs to identify COVID-19 and other viral pneumonia is acceptable (AUC in the training cohort, 0.875; AUC in the test cohort, 0.812), which is consistent with Bai et al (Accuracy $60 \%-83 \%$ ) ${ }^{[23]}$. The results of the radiologist's subjective evaluation showed that CT signs are of clinical value in identifying viral pneumonia, but there are still a lot of overlaps. Therefore, it is very challenging to distinguish two types of diseases through visual assessment. 
Radiomics is the generation of minable high throughput data through conversion of digital CT and MRI images ${ }^{[24]}$. In previous studies, radiomics had outstanding performance in the diagnosis, staging, prognosis, and treatment response prediction of tumors ${ }^{[13-15]}$. In addition, radiomics can give rise to a deeper understanding of the heterogeneity of pneumonia lesions ${ }^{[19,25,26]}$. Therefore, radiomics is theoretically a feasible method to distinguish COVID-19 pneumonia from other viral pneumonia. In our study, we selected 14 of the most predictive radiological features, and most of them were filtered or transformed first-order or texture features. It might indicate that the distinguishment between such highly imaging overlapped pneumonia may need the emphasized features in the spatial or frequency domains or the relatively higher stability of these higher-order features. In clinical cancer research, radiomics features have been shown to reflect tumor invasiveness, malignancy, and lymph node metastasis potential and other biological characteristics ${ }^{[27-29]}$. However, we speculate that the cause of CT image heterogeneity between COVID-19 and Non-COVID-19 may be different from the tumor.

Subsequently, the radiomics prediction model was constructed. The performance of the classifier in the test group was $77.8 \%$ sensitivity, $78.6 \%$ specificity, $78.2 \%$ accuracy, $77.8 \%$ positive prediction, and $78.6 \%$ negative prediction. In addition, the ROC curve was used for performance evaluation. The AUC of the test group was 0.886 , indicating good performance. In order to further improve the performance of the prediction model, we combined the radiologist's subjective visual assessment and computer radiomics features to construct the prediction model. It was found that the combined model has higher sensitivity, specificity, accuracy and AUC. And the calibration curve and decision curve showed that the reliability and stability of the combined prediction model were better.

This study has some limitations. First, as a retrospective study, there may be selection bias. But the results of our preliminary study are encouraging and will be verified in future larger studies. In addition, because of the small size of other single cases of pneumonia, we did not compare the characteristics of different viral pneumonia. Finally, the response of the lung to the virus is highly related to the host factor. CT data alone cannot completely distinguish the type of viral pneumonia, and more clinical features and laboratory examination data need to be considered. Combined with more clinical data, the predictive model may be better at identifying viral pneumonia.

In conclusion, we determined the chest CT signs and radiomics features that distinguished COVID-19 from other viral pneumonia and developed an effective predictive model. Our research shows that CT signs and radiomics features are effective tools for identifying COVID-19 and other viral pneumonia, and can assist the more precise clinical diagnosis and treatment strategy for COVID-19.

\section{Declarations}

\section{Authors' contributions}

Study concept and design, YL H and B H; acquisition of data, ZG Z, X L, YH Y, YM J, and B H; analysis and interpretation of data, YL H, YM J, B H, JL Z, and JY M; statistical analysis and drafting of the manuscript, 
YL H, ZG Z and B H; technical support, SY L.

\section{Funding}

No funding was provided for this study.

\section{Ethics approval and consent to participate}

This research was carried out in accordance with the ethical standards set up by our institutional committee. This was a retrospective study approved by our institutional review board.

\section{Consent for publication}

All authors provided consent for publication.

\section{Competing interests}

The authors had no conflicts of interest to declare in relation to this article.

\section{Acknowledgements}

The authors would like to express their appreciation for all hospital staff for their efforts to combat the COVID-19 outbreak. Thanks to all patients who participated in this study.

\section{References}

1. Zhu N, Zhang D, Wang W, Li X, Yang B, Song J, et al. A Novel Coronavirus from Patients with Pneumonia in China, 2019. N Engl J Med. 2020;382(8):727-33.

2. Guan WJ, Ni ZY, Hu Y, Liang WH, Ou CQ, He JX, et al. Clinical Characteristics of Coronavirus Disease 2019 in China. N Engl J Med. 2020. 10.1056/NEJMoa2002032. doi:10.1056/NEJMoa2002032.

3. Qiu H, Wu J, Hong L, Luo Y, Song Q, Chen D. Clinical and epidemiological features of 36 children with coronavirus disease 2019 (COVID-19) in Zhejiang, China: an observational cohort study. Lancet Infect Dis. 2020; S1473-3099(20)30198-5.

4. Chen N, Zhou M, Dong X, Qu J, Gong F, Han Y, et al. Epidemiological and clinical characteristics of 99 cases of 2019 novel coronavirus pneumonia in Wuhan, China: a descriptive study. Lancet. 2020;395(10223):507-13.

5. Fowlkes A, Steffens A, Temte J, Lonardo SD, McHugh L, Martin K, et al. Incidence of medically attended influenza during pandemic and post-pandemic seasons through the Influenza Incidence 
Surveillance Project, 2009-13. Lancet Respir Med. 2015;3(9):709-18.

6. Moriyama M, Hugentobler WJ, Iwasaki A. Seasonality of Respiratory Viral Infections. Annu Rev Virol. 2020;10.1146/annurev-virology-012420-022445.

7. Li Y, Reeves RM, Wang X, Bassat Q, Brooks WA, Cohen C, et al. Global patterns in monthly activity of influenza virus, respiratory syncytial virus, parainfluenza virus, and metapneumovirus: a systematic analysis. Lancet Glob Health. 2019;7(8):e1031-45.

8. Fang Y, Zhang H, Xie J, Lin M, Ying L, Pang P. Sensitivity of Chest CT for COVID-19: Comparison to RT-PCR. Radiology. 2020;296(2):E115-7.

9. Chung M, Bernheim A, Mei X, Zhang N, Huang M, Zeng X, et al. CT Imaging Features of 2019 Novel Coronavirus (2019-nCoV). Radiology. 2020;295(1):202-7.

10. Caruso D, Zerunian M, Polici M, Pucciarelli F, Polidori T, Rucci C, et al. Chest CT Features of COVID-19 in Rome. Italy Radiology. 2020;296(2):E79-85.

11. Koo HJ, Lim S, Choe J, Choi SH, Sung H, Do KH. Radiographic and CT Features of Viral Pneumonia. Radiographics. 2018;38(3):719-39.

12. Miller WT Jr, Mickus TJ, Barbosa E Jr, Mullin C, Van Deerlin VM, Shiley KT. CT of viral lower respiratory tract infections in adults: comparison among viral organisms and between viral and bacterial infections. AJR Am J Roentgenol. 2011;197(5):1088-95.

13. Lambin P, Leijenaar RTH, Deist TM, Peerlings J, de Jong EEC, van Timmeren J, et al. Radiomics: the bridge between medical imaging and personalized medicine. Nat Rev Clin Oncol. 2017;14(12):74962.

14. Aerts HJ, Velazquez ER, Leijenaar RT, Parmar C, Grossmann P, Carvalho S, et al. Decoding tumour phenotype by noninvasive imaging using a quantitative radiomics approach. Nat Commun. 2014;5:4006.

15. Yip SS, Aerts HJ. Applications and limitations of radiomics. Phys Med Biol. 2016;61(13):R150-66.

16. Wynants L, Van Calster B, Collins GS, Riley RD, Heinze G, Schuit E, et al. Prediction models for diagnosis and prognosis of covid-19 infection: systematic review and critical appraisal. BMJ. 2020;369:m1328.

17. Chen Y, Wang Y, Zhang Y, Zhang N, Zhao S, Zeng H, et al. A Quantitative and Radiomics approach to monitoring ARDS in COVID-19 patients based on chest CT: a retrospective cohort study. Int J Med Sci. 2020;17(12):1773-82.

18. Wu Q, Wang S, Li L, Wu Q, Qian W, Hu Y, et al. Radiomics Analysis of Computed Tomography helps predict poor prognostic outcome in COVID-19. Theranostics. 2020;10(16):7231-44.

19. Zhang T, Yuan M, Zhong Y, Zhang YD, Li H, Wu JF, et al. Differentiation of focal organising pneumonia and peripheral adenocarcinoma in solid lung lesions using thin-section CT-based radiomics. Clin Radiol. 2019;74(1):78.e23-78.e30.

20. Shi H, Han X, Jiang N, Cao Y, Alwalid O, Gu J, et al. Radiological findings from 81 patients with COVID-19 pneumonia in Wuhan, China: a descriptive study. Lancet Infect Dis. 2020; S1473- 
3099(20)30086-4.

21. van Griethuysen JJM, Fedorov A, Parmar C, Hosny A, Aucoin N, Narayan V, et al. Computational Radiomics System to Decode the Radiographic Phenotype. Cancer Res. 2017;77(21):e104-7.

22. Tang X, Du RH, Wang R, Cao TZ, Guan LL, Yang CQ, et al. Comparison of Hospitalized Patients With ARDS Caused by COVID-19 and H1N1. Chest. 2020; S0012-3692(20)30558-4.

23. Bai HX, Hsieh B, Xiong Z, Halsey K, Choi JW, Tran TML, et al. Performance of radiologists in differentiating COVID-19 from viral pneumonia on chest CT. Radiology. 2020;296(2):E46-54.

24. Lambin P, Leijenaar RTH, Deist TM, Peerlings J, de Jong EEC, van Timmeren J, et al. Radiomics: the bridge between medical imaging and personalized medicine. Nat Rev Clin Oncol. 2017;14(12):74962.

25. Wang B, Li M, Ma H, Han F, Wang Y, Zhao S, et al. Computed tomography-based predictive nomogram for differentiating primary progressive pulmonary tuberculosis from community-acquired pneumonia in children. BMC Med Imaging. 2019;19(1):63.

26. Yanling W, Duo G, Zuojun G, Zhongqiang S, Yankai W, Shan L, et al. Radiomics Nomogram Analyses for Differentiating Pneumonia and Acute Paraquat Lung Injury. Sci Rep. 2019;9(1):15029.

27. Cho HH, Lee G, Lee HY, Park H. Marginal radiomics features as imaging biomarkers for pathological invasion in lung adenocarcinoma. Eur Radiol. 2020;30(5):2984-94.

28. Takahashi S, Takahashi W, Tanaka S, Haga A, Nakamoto T, Suzuki Y, et al. Radiomics Analysis for Glioma Malignancy Evaluation Using Diffusion Kurtosis and Tensor Imaging. Int J Radiat Oncol Biol Phys. 2019;105(4):784-91.

29. Wu S, Zheng J, Li Y, Wu Z, Shi S, Huang M, et al. Development and Validation of an MRI-Based Radiomics Signature for the Preoperative Prediction of Lymph Node Metastasis in Bladder Cancer. EBioMedicine. 2018;34:76-84.

\section{Figures}


Patients with viral pneumonia in Yunnan $(n=375)$

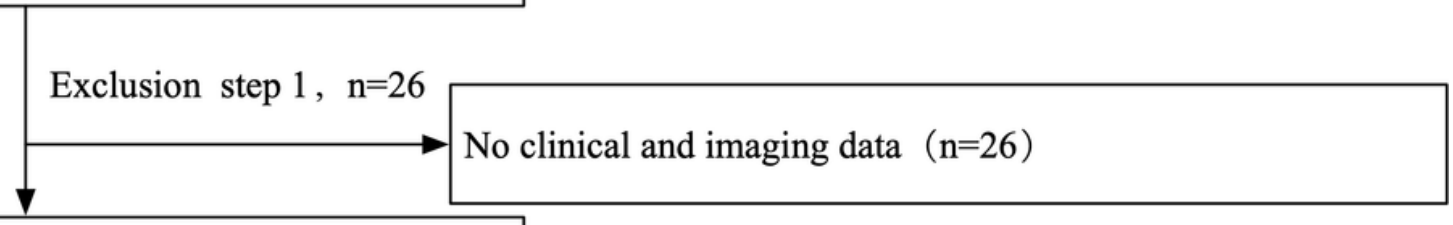

Confirmed patients with clinical and imaging data $(n=349)$

\begin{tabular}{|l|l|l|}
\hline \multirow{2}{*}{ Exclusion step 2, $\mathrm{n}=76$} & $\begin{array}{l}\text { 1. Initial chest examination were DR }(\mathrm{n}=8) \\
\text { 2. Not enough CT images }(\mathrm{n}=12) \\
\text { 3. Image format were not DICOM }(\mathrm{n}=17) \\
\text { 4. Thickness }>2 \mathrm{~mm}(\mathrm{n}=39)\end{array}$ \\
\hline
\end{tabular}

Complete clinical and CT data $(n=273)$

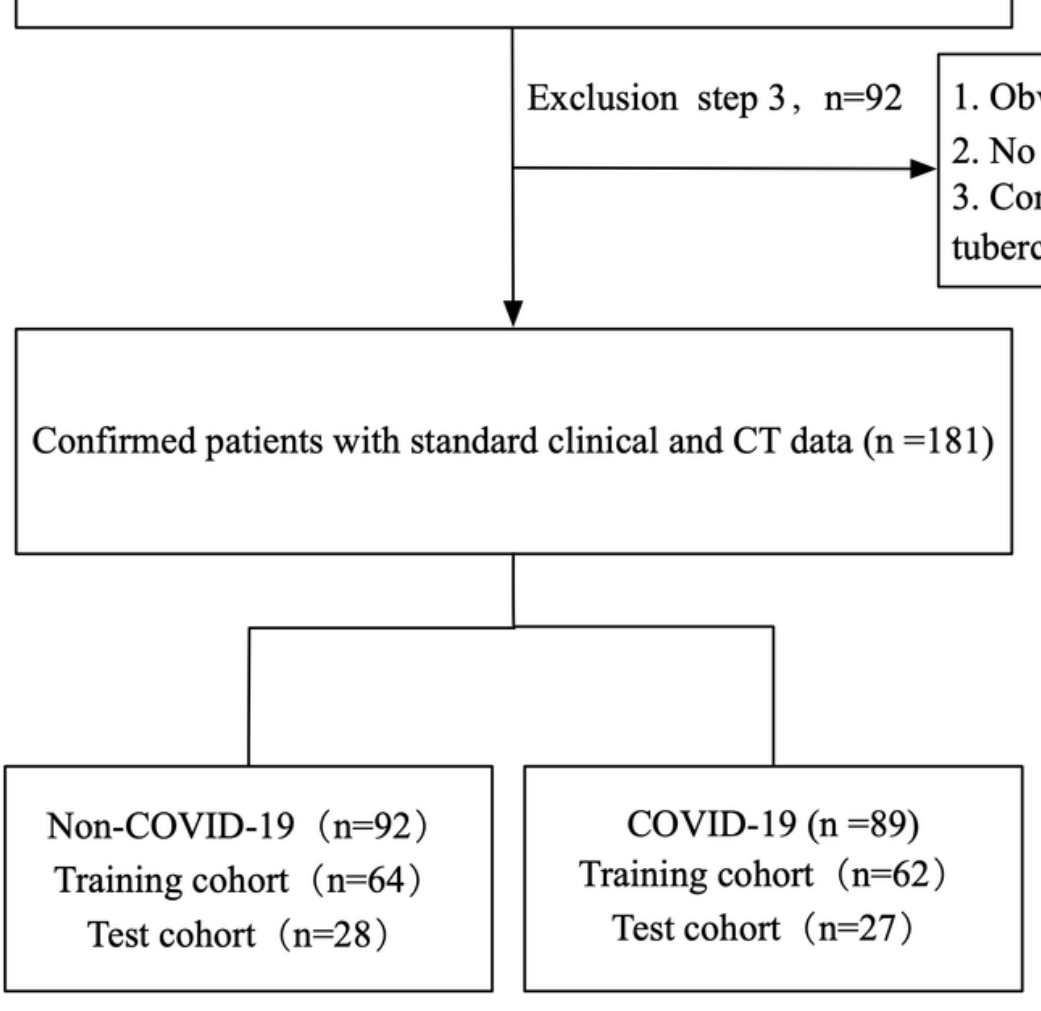

Figure 1

Flow diagram of the study design and patient enrollment in the analysis. 
log-sigma-2-0-mm-3D_firstorder_Maximum wavelet-LHH glrlm LongRunHighGrayLevelEmphasis lbp-3D-k_glszm_GrayLevelNonUniformityNormalized lbp-3 $\overline{\mathrm{D}}-\mathrm{m} 2$ _gldm_GrayLevelNonUniformity wavelet-HLL_firstorder_RobustMeanAbsoluteDeviation wavelet-HHL_glszm_GrayLevelNonUniformityNormalized lbp-3D-m2_firstorder_Median log-sigma-3-0-mm-3D glszm ZoneVariance wavelet-HH $\hat{H}_{\text {glcm }}$ InverseVariance original_shape_Sphericity

log-sigma-3-0-mm-3D firstorder Range wavelet-LLH_firstorder_Energy lbp-3D-m2_glszm_SmallAreaHighGrayLevelEmphasis wavelet-LHH_glszm_LowGrayLevelZoneEmphasis intercept

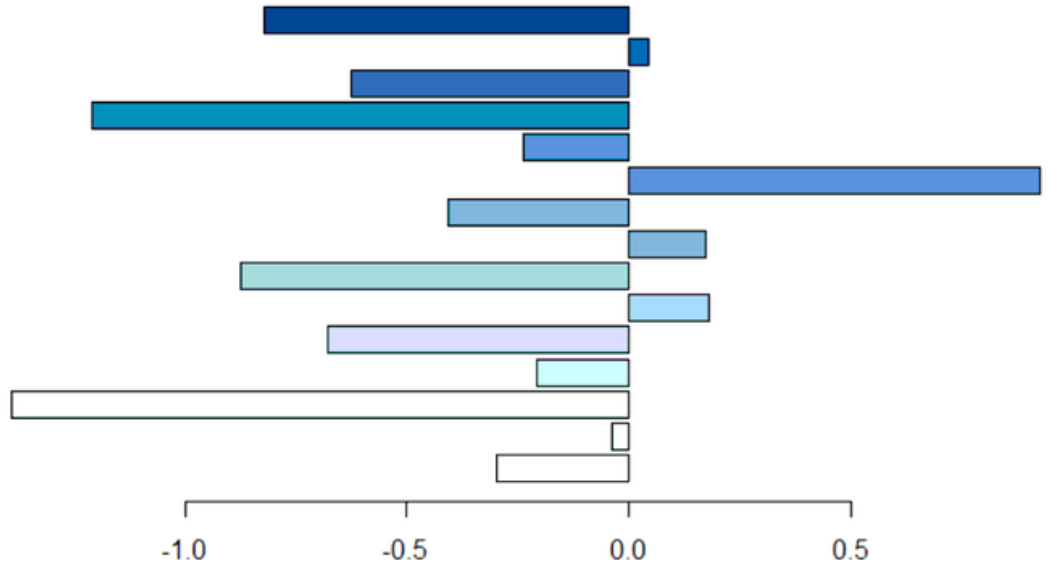

\section{Figure 2}

The selected features and their coefficient.

Points

0

10

20

30

40

50

60

70

80

90

Radscore

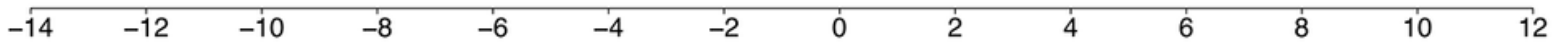

GGO

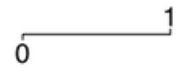

IIT

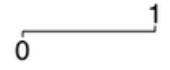

Halo

sign

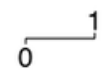

Total Points

$$
0 \quad 10
$$
20 30 40 50 60 70 80 90 100

Probability

$\begin{array}{llll}0.1 & 0.30 .50 .7 & 0.95\end{array}$

\section{Figure 3}

Nomogram based on the Radscors and CT signs for the distinction of COVID-19 pneumonia from other viral pneumonia. 0: negative, 1: positive. Radscore, radiomics score; GGO, ground-glass opacities; IIT, Intralobular interstitial thickening. 

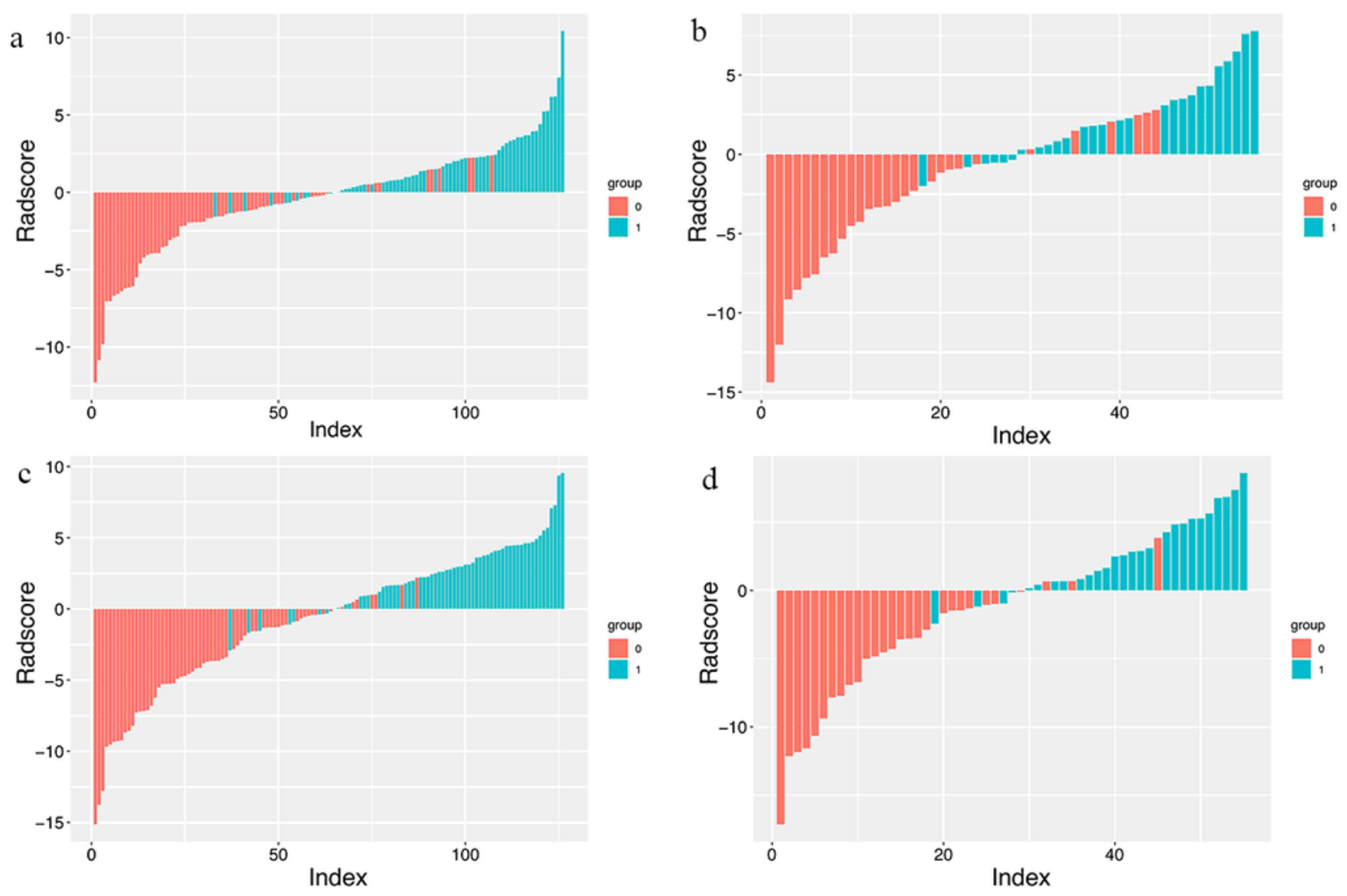

\section{Figure 4}

Radscore for patients with COVID-19 and Non-COVID-19 in the training and test cohorts. (a-b) Radscore of radiomics model in the training and test cohort respectively; (c-d) Radscore of radiomics with CT signs model in the training and test cohort respectively. 0 means Non-COVID-19, 1 means COVID-19. 

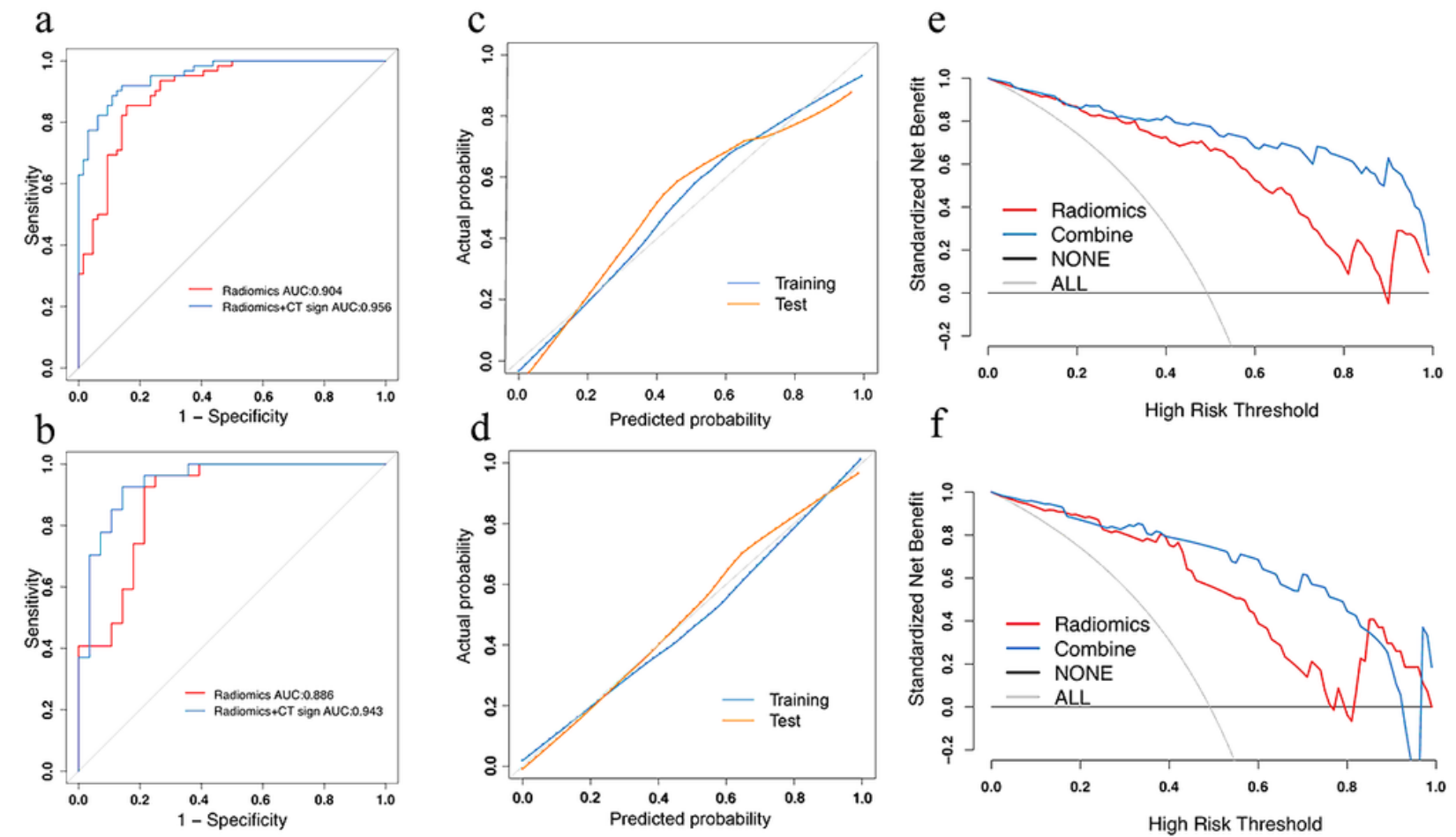

\section{Figure 5}

Predictive performance of the radiomics model and the combined model. $(a, b)$ Receiver operating characteristic (ROC) curves for the training (a) and test cohorts (b). (c, d) Calibration curves of the radiomics model (c) and the combined model (d). $(e, f)$ Decision curve analysis for two models for the training (e) and test cohorts (f).

\section{Supplementary Files}

This is a list of supplementary files associated with this preprint. Click to download.

- SupplementaryTable2.docx

- SupplementaryTable2.docx

- SupplementaryTable1.docx

- SupplementaryTable1.docx 